\title{
The Effect of Volume Fraction of Single-Walled Carbon Nanotubes on Natural Frequencies of Polymer Composite Cone-Shaped Shell Made from Poly(Methyl Methacrylate)
}

\author{
A. H. Meysami, ${ }^{1}$ H. Razavi, ${ }^{2}$ I. Fakhari Golpayegani, ${ }^{2}$ and V. Bagheri ${ }^{2}$ \\ ${ }^{1}$ Department of Metallurgy \& Material Engineering, Golpayegan University of Technology, Golpayegan 87717-65651, Iran \\ ${ }^{2}$ Department of Mechanical Engineering, Golpayegan University of Technology, Golpayegan 87717-65651, Iran
}

Correspondence should be addressed to A. H. Meysami; meysami@iust.ac.ir

Received 27 September 2016; Revised 31 December 2016; Accepted 9 January 2017; Published 14 March 2017

Academic Editor: Antonio Riveiro

Copyright (C) 2017 A. H. Meysami et al. This is an open access article distributed under the Creative Commons Attribution License, which permits unrestricted use, distribution, and reproduction in any medium, provided the original work is properly cited.

In this paper, the effect of volume fraction of single-walled carbon nanotubes on natural frequencies of polymer composite coneshaped shells made from Poly(Methyl Methacrylate) (PMMA) is studied. In order to determine the characterization of materials reinforced with nanoparticles, the molecular dynamics and mixture rule has been used. The motion equations of composite shell based on the classical thin shells theory using Hamilton's principle are obtained. Then, using the Ritz method, approximate analytical solution of the natural frequency is presented. Results indicate that the nanotubes have a noticeable effect on the natural frequencies.

\section{Introduction}

One of the most important phenomena in shells sciences is vibration due to dynamic loading, either one cyclic or impact loading. The shell and any other continuous structures have infinite natural frequencies and vibration mode shapes. If a structure vibrates with a frequency equal to its natural frequency, very little energy is needed to increase its amplitude rapidly and the structure becomes unstable. This can lead to uncomfortable consequences; for instance, the aircraft wing can be broken or the huge turbines may get out of their axis. In recent years, composite plates and shells reinforced with single-walled carbon nanotube (SWCNT) are used widely in industry. Therefore, for stability of these structures, the natural frequency should be calculated and the difference between that and the frequency of time-dependent applied loads should be determined.

Single-walled carbon nanotubes (SWCNTs) and nanocones (SWCNCs) are made of one-atom-thick carbon sheets shaped into hallow cylinders and cones. The carbon sheets give SWCNTs unique electric, chemical, and mechanical properties. In addition, extensive application of SWCNTs in nanoscale devices, NEMS, sensors, and reinforcing particles in composite materials has brought these devices into the focus of researchers' attention [1].

The mechanical studies related to SWCNTs can be divided into two general categories: (a) analysis of the static and dynamic behavior of SWCNT as a singular nanoscale part and (b) investigating the mechanical behavior of composite shells reinforced by SWCNTs. It is important to know that as dimensions are scaled down, many essential phenomena appear at the nanoscale, which are not important at macroscale. Due to size dependency of material properties in SWCNTs, it is not possible to study the dynamic behavior and mechanical properties of these components through usual methods such as classical continuum theory. Hence, the first kind can be studied through high order continuum mechanics models for nanobeam [2, 3] and for nanoshell including the nonlocal elasticity theory [4-8], the modified couple stress theory [9-13], the modified strain gradient theory [14-16], and the surface elasticity theory [17-20] and also molecular dynamic (MD) simulation [21-24] and experimental approach $[23,25]$.

The size parameter and new developed material length scale parameter, which are introduced in high order continuum theories to model the SWCNT as a singular nanoscale 
device, result in more precise estimation of natural frequencies of the nanoscale parts. Anyway, determination of Young's modulus of SWCNCs and SWCNTs [26, 27], shear modulus [27], critical load in buckling [28], and mode shapes [24] and in general determination of mechanical properties and dynamic behaviors of nanoscale structures in various shapes, sizes, and supports are the main goal of these types of analyses.

The other kind of the problems related to SWCNTs is the macroscale composites, which are reinforced by SWCNT particles. Therefore, the classical continuum theory developed for plate and shell can be used to analyze the mechanical properties and dynamic behaviors of these types of structures. One of the important issues is the effect of volume fraction of SWCNTs on the dynamic behavior of composite shells and particularly cone shaped shell of polymer composite of Poly(Methyl Methacrylate) (PMMA). PMMA is widely used in various applications for its many advantageous properties. Perhaps the most well-known of these properties is light transmission. Typical PMMA grades allow 92\% of light to pass through it, which is more than glass or other plastics. This outstanding clarity enables the use of PMMA in many different optical and related applications. PMMA will not shatter, but, it breaks into large pieces. It dissolves in most organic solvents and has poor resistance to many chemical materials. However, its environmental stability is higher than most other plastics such as polyethylene and polystyrene; therefore it is often the first choice for outdoor applications [29]. Its durability and transparency have marked it as a versatile material for a wide range of fields and applications such as skylights, sanitary ware (bathtubs), bullet proof security barriers, signs and displays, LCD screens, and furniture. Methacrylate polymers are used extensively in dental and medical applications. PMMA is compatible with human tissue, and it can be implanted in the eye when the original lens has been removed due to cataracts disease [30]. In orthopedic surgery, PMMA bone cement is used to affix implants and to remodel lost bone. Bone cement acts like a grout and not so much like a glue in arthroplasty. Although sticky, it does not bond to either the bone or the implant; it primarily fills the spaces between the prosthesis and the bone preventing motion. A major consideration when using PMMA cement is the effect of stress shielding. Since PMMA has a Young's modulus lower than that of natural bone [31], the stresses are loaded into the cement and so the bone no longer receives the mechanical signals to continue bone remodeling and so resorption will occur [32]. Nowadays, newly fabricated composites based on PMMA (whereas PMMA is used as matrix) are of high concern to the researchers. In this regard, Ke et al. [33] and Ansari et al. [34] have investigated, respectively, the nonlinear free vibration and nonlinear forced vibration behavior of nanocomposite beams reinforced with singlewalled carbon nanotube (SWCNT) based on Timoshenko beam theory. In the problem of composite plates reinforced by CNTs, Ansari et al. [35, 36] have investigated numerically the nonlinear forced vibration behavior and the geometrically nonlinear primary resonance of third-order shear deformable functionally graded CNT rectangular plates with various edge supports subjected to a harmonic excitation transverse force.
Zhang and Liew [37] have proposed an improved moving least-squares (IMLS) approximation for the field variables in the functionally graded CNT plates. They [38] also have studied that using the element-free kp-Ritz method based on first-order shear deformation theory. Ansari et al. [39] have proposed an analytical solution for the nonlinear postbuckling problem of piezoelectric FG-CNT reinforced composite cylindrical shells subjected to several loadings. They [40, 41] also have employed the variational formulation to study the buckling and vibration of axially compressed FG-CNTRC conical shells. Liew et al. [42] have studied free vibration of thin conical shells under various boundary conditions using Ritz method. Zhao and Liew [43] have studied the free vibration of conical shell panels made of functionally graded material using kp-Ritz method. Formica et al. [44] have studied the vibrational properties of composites reinforced with carbon nanotubes (CNTs), using a continuum model, based on the view of Mori et al.s theory [45, 46]. Tornabene [47] has investigated the free vibration of FGM conical and cylindrical shells. In the mentioned paper, a kind of functional material has been used which is combination of metal and ceramic. In this study, the natural frequencies of conical shells reinforced with carbon nanotubes are obtained. In order to validate the model, the results are compared with the results of isotropic case in previous research. In addition, the effect of volume fraction of nanotubes and the effect of vertex angle of the cone on the natural frequencies are investigated.

\section{Theoretical Approach}

2.1. Governing Equations of Motion. The conical shell model is shown in Figure 1. In this figure, $x$ is coordinate axis in the ridge direction of the cone, $\theta$ is the coordinate in environmental direction, and $z$ denotes the centerline of the cone. Also $(u, v, w)$ are displacements in $(x, y, z)$ coordinate systems, respectively.

2.2. Strain-Displacement Relations. Linear strain-displacement relations of the middle surface, according to Sanders' kinematic relations, are as follows:

$$
\begin{aligned}
\varepsilon_{x x}^{0} & =\frac{\partial u_{0}}{\partial x} \\
\varepsilon_{\theta \theta}^{0} & =\frac{1}{x \sin \phi} \frac{\partial v_{0}}{\partial \theta}+\frac{u_{0}}{x}+\frac{w_{0}}{x \tan \phi} \\
\gamma_{x \theta}^{0} & =\frac{\partial v_{0}}{\partial x}+\frac{1}{x \sin \phi} \frac{\partial u_{0}}{\partial \theta}-\frac{v_{0}}{x}
\end{aligned}
$$

Also, curvatures are defined as follows:

$$
\begin{aligned}
k_{x} & =\frac{\partial \psi_{x}}{\partial x} \\
k_{\theta} & =\frac{1}{x \sin \phi} \frac{\partial \psi_{\theta}}{\partial \theta}+\frac{\psi_{x}}{x} \\
\tau & =\frac{\partial \psi_{\theta}}{\partial x}+\frac{1}{x \sin \phi} \frac{\partial \psi_{x}}{\partial \theta}-\frac{\psi_{\theta}}{x}
\end{aligned}
$$



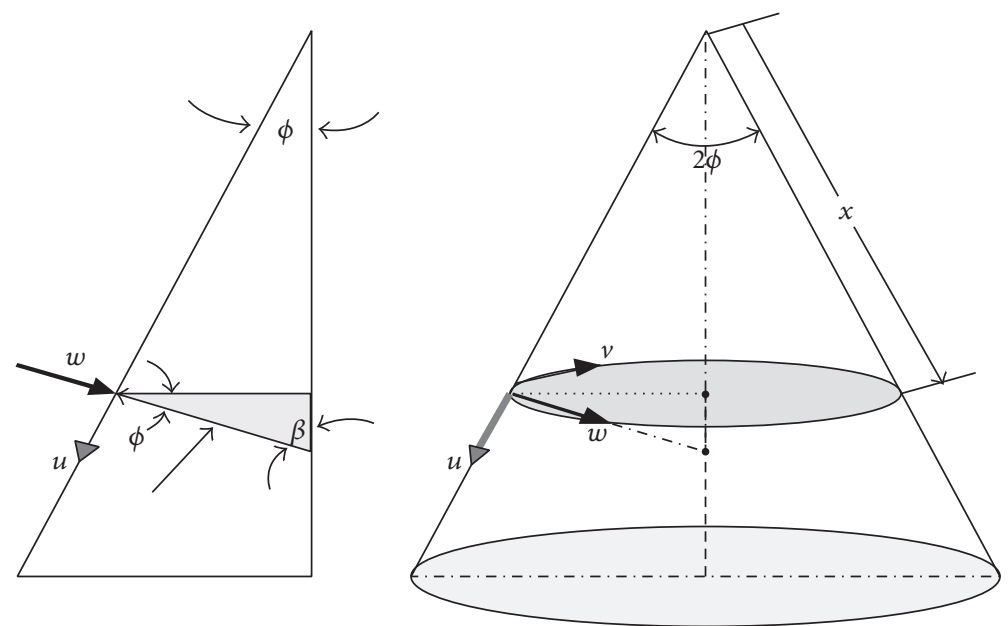

FIGURE 1: Geometry and coordinate system of a conical shell.

$$
\begin{aligned}
& \psi_{x}=\frac{-\partial w_{0}}{\partial x} \\
& \psi_{\theta}=\frac{v_{0}}{x \tan \phi}-\frac{1}{x \sin \phi} \frac{\partial w_{0}}{\partial \theta}
\end{aligned}
$$

Now, by substituting (3) in (2), the curves will be redefined as follows:

$$
\begin{aligned}
k_{x}= & \frac{\partial}{\partial x}\left(\frac{-\partial w_{0}}{\partial x}\right)=-\frac{\partial^{2} w_{0}}{\partial x^{2}}, \\
k_{\theta}= & \frac{1}{x \sin \phi} \frac{\partial}{\partial \theta}\left(\frac{+v_{0}}{x \tan \phi}-\frac{1}{x \sin \phi} \frac{\partial w_{0}}{\partial \theta}\right) \\
& +\frac{1}{x}\left(\frac{-\partial w_{0}}{\partial x}\right) \\
= & -\frac{1}{x^{2} \sin { }^{2} \phi} \frac{\partial^{2} w_{0}}{\partial \theta^{2}}+\frac{\cos \phi}{x^{2} \sin { }^{2} \phi} \frac{\partial v_{0}}{\partial \theta}-\frac{1}{x} \frac{\partial w_{0}}{\partial x} \\
\tau= & \frac{\partial}{\partial x}\left(\frac{v_{0}}{x \tan \phi}-\frac{1}{x \sin \phi} \frac{\partial w_{0}}{\partial \theta}\right) \\
& +\frac{1}{x \sin \phi} \frac{\partial}{\partial \theta}\left(\frac{-\partial w_{0}}{\partial x}\right)-\frac{v_{0}}{x^{2} \tan \phi} \\
& +\frac{1}{x^{2} \sin \phi} \frac{\partial w_{0}}{\partial \theta} \\
& +\frac{1}{x^{2} \sin \phi} \frac{\partial w_{0}}{\partial \theta} \\
& +\frac{1}{x^{2} \sin \phi} \frac{\partial w_{0}}{\partial \theta}-\frac{1}{x \sin \phi} \frac{\partial^{2} w_{0}}{\partial x \partial \theta}-\frac{1}{x^{2} \tan \phi} \\
x^{2} \tan \phi & v_{0}-\frac{\partial^{2} w_{0}}{\partial x \partial \theta}
\end{aligned}
$$

$$
\begin{aligned}
= & \frac{-2}{x \sin \phi} \frac{\partial^{2} w_{0}}{\partial x \partial \theta}+\frac{1}{x \tan \phi} \frac{\partial v_{0}}{\partial x}+\frac{2}{x^{2} \sin \phi} \frac{\partial w_{0}}{\partial \theta} \\
& -\frac{2 v_{0}}{x^{2} \tan \phi} .
\end{aligned}
$$

Strain-displacement relations for an arbitrary point in the distance $z$ from the middle surface are expressed as follows:

$$
\begin{aligned}
& \varepsilon_{x x}=\varepsilon_{x x}^{0}+z k_{x}, \\
& \varepsilon_{\theta \theta}=\varepsilon_{\theta \theta}^{0}+z k_{\theta}, \\
& \gamma_{x \theta}=\gamma_{x \theta}^{0}+z \tau .
\end{aligned}
$$

Derived equations can be expressed in matrix form as follows:

$$
\begin{aligned}
& \left\{\begin{array}{l}
\varepsilon_{x x}^{0} \\
\varepsilon_{\theta \theta}^{0} \\
\gamma_{x \theta}^{0}
\end{array}\right\}=\left\{\begin{array}{c}
\frac{\partial u_{0}}{\partial x} \\
\frac{1}{x \sin \phi} \frac{\partial v_{0}}{\partial \theta}+\frac{u_{0}}{x}+\frac{w_{0}}{x \tan \phi} \\
\frac{\partial v_{0}}{\partial x}+\frac{1}{x \sin \phi} \frac{\partial u_{0}}{\partial \theta}-\frac{v_{0}}{x}
\end{array}\right\}, \\
& \left\{\begin{array}{l}
k_{x} \\
k_{\theta} \\
\tau
\end{array}\right\} \\
& =\left\{\begin{array}{l}
-\frac{1}{x^{2} \sin ^{2} \phi} \frac{\partial^{2} w_{0}}{\partial \theta^{2}}+\frac{\cos \phi}{x^{2} \sin ^{2} \phi} \frac{\partial v_{0}}{\partial \theta}-\frac{1}{x} \frac{\partial w_{0}}{\partial x} \\
\frac{-2}{x \sin \phi} \frac{\partial^{2} w_{0}}{\partial x \partial \theta}+\frac{1}{x \tan \phi} \frac{\partial v_{0}}{\partial x}+\frac{2}{x^{2} \sin \phi} \frac{\partial w_{0}}{\partial \theta}-\frac{2 v_{0}}{x^{2} \tan \phi}
\end{array}\right\}, \\
& \left\{\begin{array}{l}
\varepsilon_{x x} \\
\varepsilon_{\theta \theta} \\
\gamma_{x \theta}
\end{array}\right\}=\left\{\begin{array}{l}
\varepsilon_{x x}^{0} \\
\varepsilon_{\theta \theta}^{0} \\
\gamma_{x \theta}^{0}
\end{array}\right\}+z\left\{\begin{array}{l}
k_{x} \\
k_{\theta} \\
\tau
\end{array}\right\} .
\end{aligned}
$$


2.3. Stress-Strain Relationships. Since the shell made of composite is reinforced with carbon nanotubes, in general it is assumed that the shells material properties are orthotropic. On the other hand, because of the carbon nanotubes setting assumptions along the ridge of the cone-shaped shell, orthotropic stress-strain will be reducible to transversely isotropic case.

$$
\left\{\begin{array}{l}
\sigma_{x x} \\
\sigma_{\theta \theta} \\
\sigma_{x \theta}
\end{array}\right\}=\left[\begin{array}{ccc}
Q_{11} & Q_{12} & 0 \\
Q_{12} & Q_{22} & 0 \\
0 & 0 & Q_{66}
\end{array}\right]\left\{\begin{array}{l}
\varepsilon_{x x} \\
\varepsilon_{\theta \theta} \\
\gamma_{x \theta}
\end{array}\right\},
$$

where

$$
\begin{aligned}
Q_{11} & =\frac{E_{1}}{1-v_{12} \nu_{21}}, \\
Q_{12} & =\frac{v_{12} E_{2}}{1-v_{12} \nu_{21}}, \\
Q_{22} & =\frac{E_{2}}{1-v_{12} \nu_{21}}, \\
Q_{66} & =G_{12} .
\end{aligned}
$$

$$
\begin{aligned}
\delta U & =\iint\left\{\int_{-h / 2}^{h / 2}\left[\sigma_{x x} \delta \varepsilon_{x x}+\sigma_{\theta \theta} \delta \varepsilon_{\theta \theta}+\sigma_{x \theta} \delta \gamma_{x \theta}\right] d z\right\} x \sin \phi d x d \theta \\
& =\iint\left\{\int_{-h / 2}^{h / 2}\left[\sigma_{x x}\left(\delta \varepsilon_{x x}^{\circ}+z \delta k_{x}\right)+\sigma_{\theta \theta}\left(\delta \varepsilon_{\theta \theta}^{\circ}+z \delta k_{\theta}\right)+\sigma_{x \theta}\left(\delta \gamma_{x \theta}^{\circ}+z \delta \tau\right)\right] d z\right\} x \sin \phi d x d \theta
\end{aligned}
$$

and the virtual work done by external loads acting upon the conical shell, $\delta V$, is defined as

$$
\delta V=-\iint q \delta w_{0} x \sin \phi d x d \theta
$$

and virtual kinetic energy, $\delta K$, is defined as
Young's modulus, shear modulus, and Poisson's ratio are obtained from the mixtures rule [50]. To simplify the equations, the following equations are considered:

$$
\left(A_{i j}, D_{i j}\right)=\int_{-h / 2}^{h / 2} Q_{i j}\left(1, z^{2}\right) d z, \quad i, j=1,2,6,
$$

where $A_{i j}$ and $D_{i j}$ are extensional and bending stiffness, respectively.

2.4. Equations of Motion. In order to derive the equations of motion, Hamilton's principle is applied:

$$
\int_{t_{1}}^{t_{2}}(\delta U+\delta V-\delta K) d t=0,
$$

where the virtual strain energy, $\delta U$, is defined as

$$
\begin{aligned}
& \int_{t_{1}}^{t_{2}}\left\{\iint\left[N_{x x} \delta \varepsilon_{x x}^{0}+M_{x x} \delta k_{x}+N_{\theta \theta} \delta \varepsilon_{\theta \theta}^{0}+M_{\theta \theta} \delta k_{\theta}+N_{x \theta} \delta \gamma_{x \theta}^{0}+M_{x \theta} \delta \tau-q \delta w_{0}-I_{0}\left(\dot{u}_{0} \delta \dot{u}_{0}+\dot{v}_{0} \delta \dot{v}_{0}+\dot{w}_{0} \delta \dot{w}_{0}\right)\right] x\right. \\
& \cdot \sin \phi d x d \theta\} d t=0 .
\end{aligned}
$$

By substituting (1) and (4) in (14), the following equation can be obtained:

$$
\begin{aligned}
& \int_{t_{2}}^{t_{1}}\left\{\int \int \left[N_{x x} \frac{\partial \delta u_{0}}{\partial x}-M_{x x} \frac{\partial^{2} \delta w_{0}}{\partial x^{2}}\right.\right. \\
& +N_{\theta \theta}\left(\frac{1}{x \sin \phi} \frac{\partial \delta v_{0}}{\partial \theta}+\frac{\delta u_{0}}{x}+\frac{\delta w_{0}}{x \tan \phi}\right) \\
& +M_{\theta \theta}\left(\frac{-1}{x^{2} \sin ^{2} \phi} \frac{\partial^{2} \delta w_{0}}{\partial \theta^{2}}+\frac{\cos \phi}{x^{2} \sin ^{2} \phi} \frac{\partial \delta v_{0}}{\partial \theta}-\frac{1}{x} \frac{\partial \delta w_{0}}{\partial x}\right)
\end{aligned}
$$

$$
\delta K=\iiint_{-h / 2}^{h / 2} \rho\left[\dot{u}_{0} \delta \dot{u}_{0}+\dot{v}_{0} \delta \dot{v}_{0}+\dot{w}_{0} \delta \dot{w}_{0}\right] x
$$

$\cdot \sin \phi d z d x d \theta$.

Substituting $\delta U$ in the Hamilton principle (10) and then integrating along the thickness, $\delta V$ and $\delta K$ yield 
where stress resultants are defined as follows:

$$
\begin{aligned}
& N_{i j}=\int_{-h / 2}^{h / 2} \sigma_{i j} d z, \\
& M_{\ddot{y}}=\int_{-h / 2}^{h / 2} \sigma_{i j} z d z,
\end{aligned}
$$

$$
i j=x x, \theta \theta, x \theta \text {. }
$$

Also,

$$
I_{0}=\int_{-h / 2}^{h / 2} \rho d z
$$

Now, applying integration by parts yields the following equations of motion:

$$
\begin{array}{r}
\frac{\partial N_{x x}}{\partial x}+\frac{N_{x x}-N_{\theta \theta}}{x}+\frac{1}{x \sin \phi} \frac{\partial N_{x \theta}}{\partial \theta}=I_{0} \ddot{u}_{0} \\
\frac{1}{x \sin \phi} \frac{\partial N_{\theta \theta}}{\partial \theta}+\frac{\partial N_{x \theta}}{\partial x}+\frac{2 N_{x \theta}}{x}+\frac{Q_{\theta}}{x \tan \phi}=I_{0} \ddot{v}_{0} \\
-\left(\frac{N_{\theta \theta}}{x \tan \phi}\right)+\frac{\partial Q_{x}}{\partial x}+\frac{Q_{x}}{x}+\frac{1}{x \sin \phi} \frac{\partial Q_{\theta}}{\partial \theta}+q=I_{0} \ddot{w}_{0},
\end{array}
$$

where

$$
\begin{aligned}
& Q_{x}=\frac{M_{x x}}{x}+\frac{\partial M_{x x}}{\partial x}+\frac{1}{x \sin \phi} \frac{\partial M_{x \theta}}{\partial \theta}-\frac{M_{\theta \theta}}{x}, \\
& Q_{\theta}=\frac{1}{x \sin \phi} \frac{\partial M_{\theta \theta}}{\partial \theta}+\frac{\partial M_{x \theta}}{\partial x}+\frac{2 M_{x \theta}}{x} .
\end{aligned}
$$

To express the equations of motion in terms of displacement, stress resultants versus displacement should be derived as follows:

$$
\begin{aligned}
& N_{x x}=\int_{-h / 2}^{h / 2} \sigma_{x x} d z=\int_{-h / 2}^{h / 2}\left(Q_{11} \varepsilon_{x x}+Q_{12} \varepsilon_{\theta \theta}\right) d z \\
& =\int_{-h / 2}^{h / 2}\left[\left(Q_{11} \varepsilon_{x x}^{0}+Q_{12} \varepsilon_{\theta \theta}^{0}\right)+z\left(Q_{11} k_{x}+Q_{12} k_{\theta}\right)\right] d z \\
& =\int_{-h / 2}^{h / 2}\left(Q_{11} \varepsilon_{x x}^{0}+Q_{12} \varepsilon_{\theta \theta}^{0}\right) d z=A_{11} \varepsilon_{x x}^{0}+A_{12} \varepsilon_{\theta \theta}^{0} .
\end{aligned}
$$

Similarly, the other stress resultants are obtained which can be represented in matrix form as follows:

$$
\begin{aligned}
& \left\{\begin{array}{l}
N_{x x} \\
N_{\theta \theta} \\
N_{x \theta} \\
M_{x x} \\
M_{\theta \theta} \\
M_{x \theta}
\end{array}\right\}=\left[\begin{array}{cccccc}
A_{11} & A_{12} & 0 & 0 & 0 & 0 \\
A_{12} & A_{22} & 0 & 0 & 0 & 0 \\
0 & 0 & A_{66} & 0 & 0 & 0 \\
0 & 0 & 0 & D_{11} & D_{12} & 0 \\
0 & 0 & 0 & D_{12} & D_{22} & 0 \\
0 & 0 & 0 & 0 & 0 & D_{66}
\end{array}\right]\left\{\begin{array}{c}
\varepsilon_{x x}^{0} \\
\varepsilon_{\theta \theta}^{0} \\
\gamma_{x \theta}^{0} \\
k_{x} \\
k_{\theta} \\
\tau
\end{array}\right\}, \\
& \left\{\begin{array}{l}
N_{x x} \\
N_{\theta \theta} \\
N_{x \theta}
\end{array}\right\}=\left[\begin{array}{ccc}
A_{11} & A_{12} & 0 \\
A_{12} & A_{22} & 0 \\
0 & 0 & A_{66}
\end{array}\right]\left\{\begin{array}{c}
\frac{\partial u_{0}}{\partial x} \\
\frac{1}{x \sin \phi} \frac{\partial v_{0}}{\partial \theta}+\frac{u_{0}}{x}+\frac{w_{0}}{x \tan \phi} \\
\frac{\partial v_{0}}{\partial x}+\frac{1}{x \sin \phi} \frac{\partial u_{0}}{\partial \theta}-\frac{v_{0}}{x}
\end{array}\right\}, \\
& \left\{\begin{array}{l}
M_{x x} \\
M_{\theta \theta} \\
M_{x \theta}
\end{array}\right\}=\left[\begin{array}{ccc}
D_{11} & D_{12} & 0 \\
D_{12} & D_{22} & 0 \\
0 & 0 & D_{66}
\end{array}\right]\left[\begin{array}{c}
-\frac{\partial^{2} w_{0}}{\partial x^{2}} \\
-\frac{1}{x^{2} \sin ^{2} \phi} \frac{\partial^{2} w_{0}}{\partial \theta^{2}}+\frac{\cos \phi}{x^{2} \sin ^{2} \phi} \frac{\partial v_{0}}{\partial \theta}-\frac{1}{x} \frac{\partial w_{0}}{\partial x} \\
\frac{-2}{x \sin \phi} \frac{\partial^{2} w_{0}}{\partial x \partial \theta}+\frac{1}{x \tan \phi} \frac{\partial v_{0}}{\partial x}+\frac{2}{x^{2} \sin \phi} \frac{\partial w_{0}}{\partial \theta}-\frac{2 v_{0}}{x^{2} \tan \phi}
\end{array}\right] .
\end{aligned}
$$

2.5. Energy Functional. For the conical shell, the integral expressions of strain and kinetic energies are as follows:

$$
U=\frac{1}{2} \iint\left\{\int_{-h / 2}^{h / 2}\left[\sigma_{x x} \varepsilon_{x x}+\sigma_{\theta \theta} \delta \varepsilon_{\theta \theta}+\sigma_{x \theta} \gamma_{x \theta}\right] d z\right\} x
$$

$\cdot \sin \phi d x d \theta$

$$
K=\frac{1}{2} \iiint_{-h / 2}^{h / 2} \rho\left[\dot{u}_{0}^{2}+\dot{v}_{0}^{2}+\dot{w}_{0}^{2}\right] x \sin \phi d z d x d \theta .
$$

The above equations can be rewritten as follows: 


$$
\begin{aligned}
& U=\frac{1}{2} \iint\left\{\left[\int_{-h / 2}^{h / 2}\left[\sigma_{x x}\left(\varepsilon_{x x}^{0}+z k_{x}\right)+\sigma_{\theta \theta}\left(\varepsilon_{\theta \theta}^{0}+z k_{\theta}\right)+\sigma_{x \theta}\left(\gamma_{x \theta}^{0}+z \tau\right)\right] d z\right\} x \sin \phi d x d \theta,\right. \\
& U=\frac{1}{2} \iint\left[N_{x x} \varepsilon_{x x}^{0}+M_{x x} k_{x}+N_{\theta \theta} \varepsilon_{x x}^{0}+M_{\theta \theta} k_{\theta}+N_{x \theta} \gamma_{x \theta}^{0}+M_{x \theta} \tau\right] x \sin \phi d x d \theta, \\
& K=\frac{1}{2} \iiint\left(\rho\left[\dot{u}^{2}+\dot{v}^{2}+\dot{w}^{2}\right]\right) x \sin \phi d z d x d \theta=\frac{1}{2} I_{0} \int_{0}^{2 \pi} \int_{0}^{L}\left[\dot{u}_{0}^{2}+\dot{v}_{0}^{2}+\dot{w}_{0}^{2}\right] x \sin \phi d x d \theta .
\end{aligned}
$$

Therefore, the energy functional, depending on the strain and kinetic energy, is expressed as follows:

$$
\Pi=K-U
$$

2.6. Formulation of the Eigen Value Problem. Removing harmonic component and insertion of the displacement function in (24), the maximum energy functional is expressed as follows:

$$
\widehat{\Pi}=K_{\max }-U_{\max }
$$

where $U_{\max }$ and $K_{\max }$ are the maximum strain energy and maximum kinetic energy, respectively. By minimizing the functional equation (25) with respect to the available coefficients, an eigen value equation is obtained as follows:

$$
\begin{aligned}
& \frac{\partial \widehat{\Pi}}{\partial a_{i}}=0 ; \quad i=1,2,3 \Longrightarrow \\
& {\left[\begin{array}{ccc}
K_{11}-\omega^{2} M_{11} & K_{12} & K_{13} \\
K_{21} & K_{22}-\omega^{2} M_{22} & K_{23} \\
K_{31} & K_{32} & K_{33}-\omega^{2} M_{33}
\end{array}\right]\left\{\begin{array}{l}
a_{1} \\
a_{2} \\
a_{3}
\end{array}\right\}} \\
& \quad=\left\{\begin{array}{l}
0 \\
0 \\
0
\end{array}\right\} .
\end{aligned}
$$

To obtain the natural frequency of conical shape composite shell reinforced with nanotubes, it is sufficient to put the determinant of coefficients matrix equal to zero.

$$
\left|\begin{array}{ccc}
K_{11}-\omega^{2} M_{11} & K_{12} & K_{13} \\
K_{21} & K_{22}-\omega^{2} M_{22} & K_{23} \\
K_{31} & K_{32} & K_{33}-\omega^{2} M_{33}
\end{array}\right|=0 .
$$

2.7. The Expression of Displacement Functions. To obtain the strain and kinetic energies, first the displacement functions should be determined. It should be noted that these functions must satisfy the boundary conditions. Conical shell that will be investigated here is a complete conical shell with a fixed base. Consequently, the boundary conditions can be expressed as follows:

$$
\begin{aligned}
& u_{0}=v_{0}=w_{0}=\frac{\partial w_{0}}{\partial x}=0, \quad \text { at } x=0, \\
& u_{0}=v_{0}=w_{0}=\frac{\partial w_{0}}{\partial x}=0, \quad \text { at } x=L .
\end{aligned}
$$

According to the mentioned study, the authors will use the following displacement functions to provide an approximate analytical solution based on the Ritz method:

$$
\begin{aligned}
& u_{0}=a_{1} x^{2}(x-L)^{2} \cos n \theta \cos \omega t, \\
& v_{0}=a_{2} x^{2}(x-L)^{2} \sin n \theta \cos \omega t, \\
& w_{0}=a_{3} x^{2}(x-L)^{2} \cos n \theta \cos \omega t
\end{aligned}
$$

2.8. Mixtures Rule. Suppose that the composite shell that has been strengthened with carbon nanotubes uniformly along the $x$ coordinate is made of a mixture of single-walled carbon nanotubes, which is assumed to be isotropic. Here, the main problem is determining the effective material properties of composites reinforced with carbon nanotubes. The effective physical properties can be estimated using the Mori-Tanaka model or mixtures rule $[45,50]$. According to the rule of mixtures, Young's moduli and shear moduli can be expressed as follows:

$$
\begin{aligned}
& E_{11}=\eta_{1} V_{\mathrm{CN}} E_{11}^{\mathrm{CN}}+V_{m} E^{m}, \\
& \frac{\eta_{2}}{E_{22}}=\frac{V_{\mathrm{CN}}}{E_{22}^{\mathrm{CN}}}+\frac{V_{m}}{E^{m}}, \\
& \frac{\eta_{3}}{G_{12}}=\frac{V_{\mathrm{CN}}}{G_{12}^{\mathrm{CN}}}+\frac{V_{m}}{G^{m}},
\end{aligned}
$$

where $E_{11}^{\mathrm{CN}}, E_{22}^{\mathrm{CN}}$, and $G_{12}^{\mathrm{CN}}$ are Young's and shear moduli of carbon nanotubes, respectively. $E^{m}$ and $G^{m}$ are the corresponding properties, related to the background. $\eta_{j}$ is the efficiency parameters of carbon nanotubes which is determined by adaptation of elastic moduli from molecular dynamics simulation results and the mixtures rules. $V_{\mathrm{CN}}$ and $V_{m}$ are volume fractions of carbon nanotubes and background, which are related as follows:

$$
V_{\mathrm{CN}}+V_{m}=1
$$


TABLE 1: Temperature-dependent material properties of single-walled carbon nanotubes [48].

\begin{tabular}{lccccc}
\hline Temperature $(\mathrm{K})$ & $E_{11}^{\mathrm{CN}}(\mathrm{TPa})$ & $E_{22}^{\mathrm{CN}}(\mathrm{TPa})$ & $G_{12}^{\mathrm{CN}}(\mathrm{TPa})$ & $\alpha_{11}^{\mathrm{CN}}\left(\times 10^{-6} / \mathrm{K}\right)$ & $\alpha_{22}^{\mathrm{CN}}\left(\times 10^{-6} / \mathrm{K}\right)$ \\
\hline 300 & 5.6466 & 7.0800 & 1.9445 & 3.4584 & 5.1682 \\
\hline
\end{tabular}

TABLE 2: Young's moduli for the composite PMMA/CNT: mixtures rule in comparison with molecular dynamics.

\begin{tabular}{lccccc}
\hline \multirow{2}{*}{$V_{\mathrm{CN}}^{*}$} & \multicolumn{2}{c}{ Molecular dynamics [49] } & \multicolumn{2}{c}{ Mixtures rule [49, 50] } & $\eta_{1}$ \\
\hline 0.12 & $E_{11}$ & $E_{22}$ & 94.78 & 0.137 & 2.9 \\
0.17 & 94.6 & 2.9 & 138.68 & 0.142 & 4.9 \\
0.28 & 138.9 & 4.9 & 224.50 & 0.141 & 5.5 \\
\hline
\end{tabular}

Suppose the volume fraction, $V_{\mathrm{CN}}$, as follows:

$$
V_{\mathrm{CN}}=w(z) V_{\mathrm{CN}}^{*}
$$

where

$$
V_{\mathrm{CN}}^{*}=\frac{w_{\mathrm{CN}}}{w_{\mathrm{CN}}+\left(\rho_{\mathrm{CN}} / \rho_{m}\right)-\left(\rho_{\mathrm{CN}} / \rho_{m}\right) w_{\mathrm{CN}}}
$$

where $w_{\mathrm{CN}}$ is the mass fraction of carbon nanotubes. For a uniform distribution of nanotubes, it is considered that $V_{\mathrm{CN}}=V_{\mathrm{CN}}^{*}$. Poisson's ratio and mass density can be calculated as follows:

$$
\begin{gathered}
v_{12}=V_{\mathrm{CN}}^{*} \nu_{12}^{\mathrm{CN}}+V_{m} \nu^{m}, \\
\rho=V_{\mathrm{CN}}^{*} \rho^{\mathrm{CN}}+V_{m} \rho^{m} .
\end{gathered}
$$

Consequently, according to (9), $A_{i j}$ stiffness equations for a uniform distribution of nanotubes will be obtained as follows:

$$
\begin{aligned}
& A_{11}=\frac{1}{1-v_{12} v_{21}}\left[V_{\mathrm{CN}}^{*}\left(\eta_{1} E_{11}^{\mathrm{CN}}-E^{m}\right)+E^{m}\right] h, \\
& A_{12}=\frac{v_{12}}{1-v_{12} v_{21}}\left[\frac{\eta_{2} E_{22}^{\mathrm{CN}} E^{m}}{E_{22}^{\mathrm{CN}}+\left(E^{m}-E_{22}^{\mathrm{CN}}\right) V_{\mathrm{CN}}^{*}}\right] h, \\
& A_{22}=\frac{1}{1-v_{12} \nu_{21}}\left[\frac{\eta_{2} E_{22}^{\mathrm{CN}} E^{m}}{E_{22}^{\mathrm{CN}}+\left(E^{m}-E_{22}^{\mathrm{CN}}\right) V_{\mathrm{CN}}^{*}}\right] h, \\
& A_{66}=\left[\frac{\eta_{3} G_{12}^{\mathrm{CN}} G^{m}}{G_{12}^{\mathrm{CN}}+\left(G^{m}-G_{12}^{\mathrm{CN}}\right) V_{\mathrm{CN}}^{*}}\right] h .
\end{aligned}
$$

According to the above equations, for a uniform distribution of nanotubes, there is $D_{i j}=\left(h^{2} / 12\right) A_{i j}$; also

$$
I_{0}=\left[V_{\mathrm{CN}}^{*}\left(\rho^{\mathrm{CN}}-\rho^{m}\right)+\rho^{m}\right] h .
$$

\section{Material Properties of Carbon Nanotubes}

Based on the results of molecular dynamics simulations, size and temperature dependent material properties of singlewalled carbon nanotubes [48] can be obtained numerically as in Table 1.
3.1. Material Properties of PMMA Polymer. The material properties of considered background in temperature equal to $300^{\circ} \mathrm{K}$ are assumed as follows:

$$
\begin{aligned}
v^{m} & =0.34, \\
\rho^{m} & =1.15 \mathrm{~g} / \mathrm{cm}^{3}, \\
E^{m} & =2.5 \mathrm{GPa}, \\
G^{m} & =\frac{E^{m}}{2\left(1+v^{m}\right)}=0.933 \mathrm{Gpa} .
\end{aligned}
$$

For short fiber composites $\eta_{1}$ is usually considered to be 0.2 . However, there is no experimental method for determining the value of $\eta_{j}$. Here, with adaption of Young's moduli $E_{11}$ and $E_{22}$ obtained from the mixtures rule and molecular dynamics, efficiency parameters $\eta_{1}$ and $\eta_{2}$ are estimated. Comparison of the results shows that if the efficiency parameters are chosen properly, Young's modulus, obtained from molecular dynamics, is well adapted to mixtures rule results, as shown in Table 2.

\section{Results and Validation}

It should be noted that there are various shell models according to the thickness of the shell and various models can be used for the variable density of filling the polymer with nanotubes. In general the vibration solution for polymer composite cone-shaped shell made from PMMA is hard. However, in this section, thin shells model with uniform distribution of nanotubes in composite cone-shaped shell has been used; therefore results are extracted for special case. Validation of current study has been undertaken with [44, 51]. In this case, the parameter $h_{2} / 12 R_{2}^{2}$ is considered to be equal to $10^{-5}$ for the conical shell. It is considered in Table 3 that the errors of the present results in comparison with the solution presented in [51] are less than $1 \%$ for every vertex angle.

4.1. Numerical Results of the PMMA/CNT Composite. In this section, by considering the fixed PMMA/CNT composite shell, the numerical results of the nondimensional frequencies with respect to the different volume fractions 
TABLE 3: Nondimensional frequency of a fixed conical shell with a fixed vertex.

\begin{tabular}{|c|c|c|c|}
\hline \multirow[b]{3}{*}{$\beta$} & \multicolumn{3}{|c|}{$n$} \\
\hline & 0 & 1 & 2 \\
\hline & $\begin{array}{c}\text { Leisa } \\
\text { (presented } \\
\text { results) }\end{array}$ & $\begin{array}{c}\text { Leisa } \\
\text { (presented } \\
\text { results) }\end{array}$ & $\begin{array}{c}\text { Leisa } \\
\text { (presented } \\
\text { results) }\end{array}$ \\
\hline 15 & 0.223180 .2231 & 0.15410 .1541 & 0.8010 .801 \\
\hline$\%$ diff & 0.01165 & 0.00 & 0.00 \\
\hline 30 & 0.80030 .8004 & 0.49380 .4938 & 0.21590 .2157 \\
\hline$\%$ diff & 0.00975 & 0.00 & 0.0764 \\
\hline 45 & 1.50141 .5014 & 0.72560 .7255 & 0.27660 .2764 \\
\hline$\%$ diff & 0.00533 & 0.12277 & 0.0767 \\
\hline 60 & 1.76591 .7660 & 0.54500 .5449 & 0.19270 .1926 \\
\hline$\%$ diff & 0.00113 & 0.01 & 0.0745 \\
\hline
\end{tabular}

TABLE 4: Nondimensional frequency variation of fixed PMMA/CNT composite shell for $V_{\mathrm{CN}}^{*}=0$.

\begin{tabular}{|c|c|c|c|c|c|c|}
\hline \multirow{2}{*}{$\beta$} & \multicolumn{6}{|c|}{$n$} \\
\hline & 0 & 1 & 2 & 3 & 4 & 5 \\
\hline 15 & 0.223141 & 0.126679 & 0.05998 & 0.060032 & 0.121503 & 0.269507 \\
\hline 30 & 0.800276 & 0.38597 & 0.15421 & 0.100899 & 0.140962 & 0.277957 \\
\hline 45 & 1.50132 & 0.526649 & 0.193304 & 0.115026 & 0.145165 & 0.27607 \\
\hline 60 & 1.7659 & 0.374958 & 0.133334 & 0.085712 & 0.126232 & 0.260175 \\
\hline 75 & 0.739415 & 0.105912 & 0.04092 & 0.042762 & 0.100341 & 0.240933 \\
\hline
\end{tabular}

of carbon nanotubes, different values of $\beta$, and various $V_{\mathrm{CN}}^{*}$ are presented in Tables 4, 5 and 6 . In addition, frequency variations are plotted with respect to the number of modes " $n$ " in Figures 2, 3, and 4 for specific volume fractions of PMMA/CNT composite shell. The figures show that the most important effects of using nanotubes are in elementary modes of PMMA/CNT composite shell. Also, for " $n$ ” greater than 3 , the frequencies obtained for different $\beta$ tend to converge. It can be concluded that values of nondimensional frequencies in aforementioned range do not depend on the angle of cone apexes and the volume fraction.

\section{Conclusion}

In this paper, the effect of volume fraction of single-walled carbon nanotubes on natural frequencies of cone-shaped polymer composite shells made of PMMA is studied. There are various shell models according to the thickness of the shell and various models can be used for the variable density of filling the polymer with nanotubes. In general the vibration solution for polymer composite cone-shaped shell made from PMMA is hard. However, a complete conical shell with a fixed base is investigated. Equations of motion were developed based on Hamilton's principle. Thin shells model with uniform distribution of nanotubes in composite cone-shaped shell has been used; therefore results are extracted for special case. Regardless of the volume fraction amount of nanotubes, it is observed that the greatest decrease in nondimensional frequency parameter with increasing " $n$ " parameter occurs in

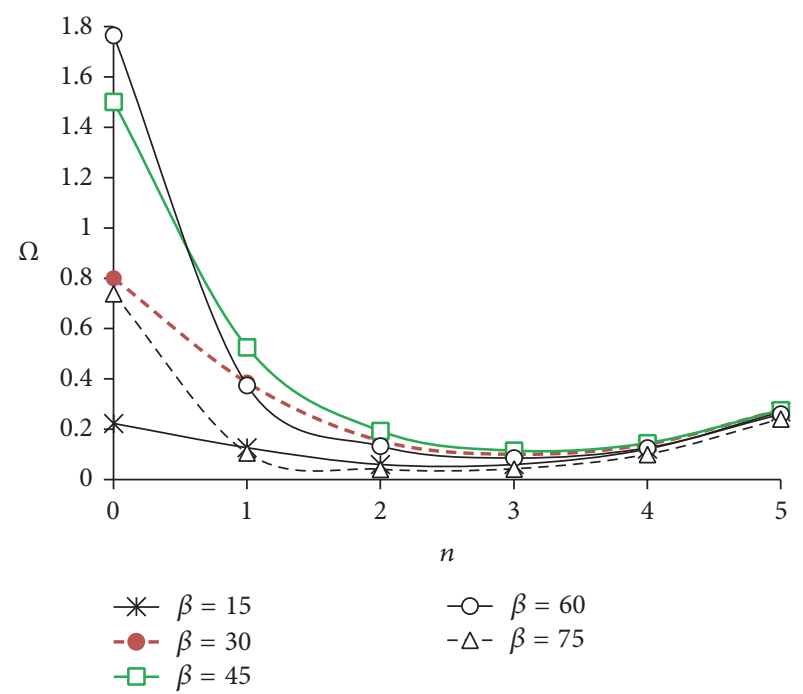

FIgURE 2: Nondimensional frequency variation of fixed PMMA/ CNT composite shell for $V_{\mathrm{CN}}^{*}=0$.

the first modes. In some cases, this reduction has increased up to 4 times. In addition, for number of modes " $n$ " greater than 3 , the frequencies obtained for different angle of cone apexes tend to converge. It is considered that the errors of the present results in comparison with the solution presented by Leisa are less than $1 \%$ for every vertex angle. It is concluded that values of nondimensional frequencies in aforementioned range do 
TABLE 5: Nondimensional frequency variation of fixed PMMA/CNT composite shell for $V_{\mathrm{CN}}^{*}=0.12$.

\begin{tabular}{|c|c|c|c|c|c|c|}
\hline \multirow{2}{*}{$\beta$} & \multicolumn{6}{|c|}{$n$} \\
\hline & 0 & 1 & 2 & 3 & 4 & 5 \\
\hline$\overline{15}$ & 0.444175 & 0.281997 & 0.203815 & 0.203427 & 0.264869 & 0.41323 \\
\hline 30 & 1.21041 & 0.517539 & 0.258945 & 0.203185 & 0.241179 & 0.376852 \\
\hline 45 & 2.27495 & 0.61614 & 0.260705 & 0.175626 & 0.200352 & 0.327925 \\
\hline 60 & 3.3595 & 0.433397 & 0.174726 & 0.115444 & 0.149108 & 0.279531 \\
\hline 75 & 4.15994 & 0.136299 & 0.055915 & 0.050495 & 0.105159 & 0.244842 \\
\hline
\end{tabular}

TABLE 6: Nondimensional frequency variation of fixed PMMA/CNT composite shell for $V_{\mathrm{CN}}^{*}=0.28$.

\begin{tabular}{|c|c|c|c|c|c|c|}
\hline \multirow{2}{*}{$\beta$} & \multicolumn{6}{|c|}{$n$} \\
\hline & 0 & 1 & 2 & 3 & 4 & 5 \\
\hline 15 & 0.880079 & 0.580785 & 0.43786 & 0.437286 & 0.549704 & 0.821152 \\
\hline 30 & 2.26357 & 0.986998 & 0.514793 & 0.413378 & 0.483145 & 0.731539 \\
\hline 45 & 4.19439 & 1.14393 & 0.494933 & 0.339869 & 0.385371 & 0.618985 \\
\hline 60 & 6.17178 & 0.797481 & 0.324699 & 0.21642 & 0.278094 & 0.51685 \\
\hline 75 & 7.64184 & 0.250454 & 0.103402 & 0.093363 & 0.193304 & 0.448963 \\
\hline
\end{tabular}

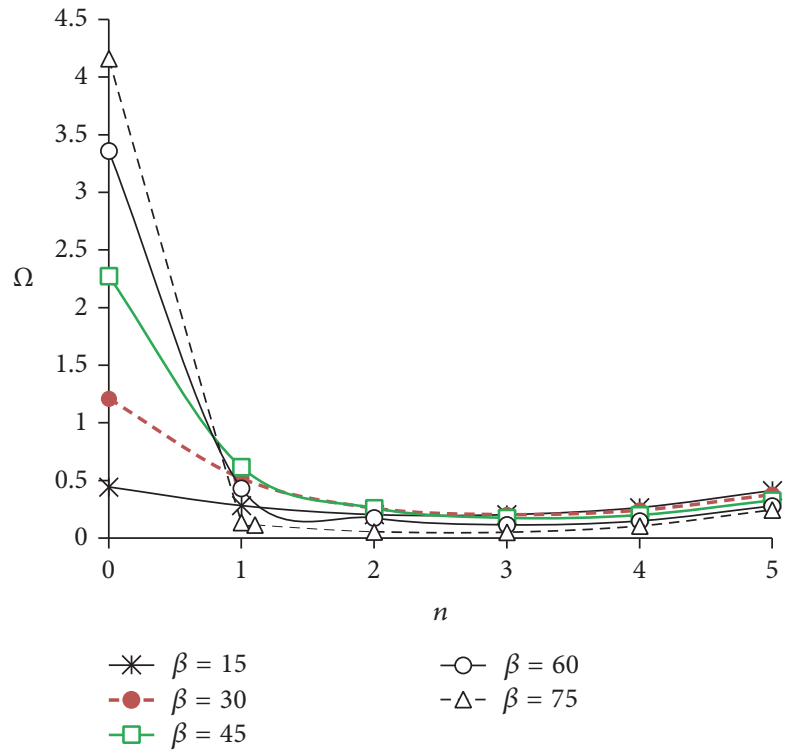

FIGURE 3: Nondimensional frequency variation of fixed PMMA/ CNT composite shell for $V_{\mathrm{CN}}^{*}=0.12$.

not depend on the angle of the cone apexes and the volume fraction.

\section{Nomenclature}

$a_{i}: \quad$ Ritz approximation coefficients

$A_{i j}$ : Extensional stiffness for a uniform distribution of nanotubes

$D_{i j}$ : Bending stiffness for a uniform distribution of nanotubes

$E_{i j}$ : Young's moduli

$E_{i j}^{\mathrm{CN}}$ : Young's moduli of carbon nanotubes

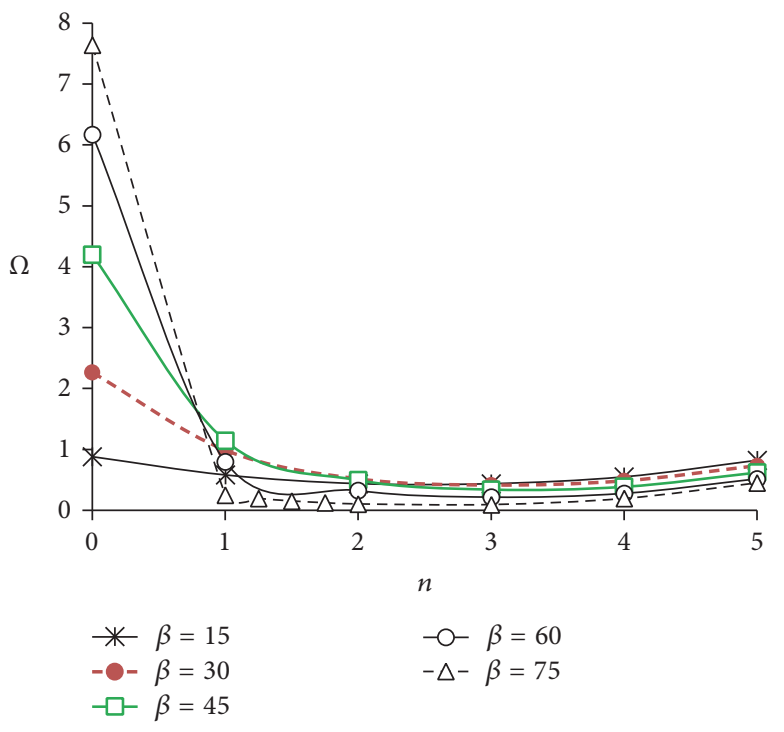

FIGURE 4: Nondimensional frequency variation of fixed PMMA/ CNT composite shell for $V_{\mathrm{CN}}^{*}=0.28$.

$E^{m}:$

$G_{i j}$ :

$G_{i j}^{\mathrm{CN}}$ :

$G^{m}$ :

$h:$

$I_{0}:$

$K$ : Virtual kinetic energy

$K_{i j}: \quad$ Matrix components

$k_{x}, k_{\theta}, \tau: \quad$ Curvature parameters

$K_{\text {max }}$ : Maximum kinetic energy

$L: \quad$ Length of the cone

$M_{i j}$ : Moment resultant

$M_{x x}, M_{x \theta}, M_{\theta \theta}$ : Moment resultant components 


\begin{tabular}{|c|c|}
\hline$n:$ & Mode number \\
\hline$N_{i j}:$ & Force resultant \\
\hline$N_{x x}, N_{x \theta}, N_{\theta \theta}:$ & Force resultant components \\
\hline$Q_{i j}:$ & Orthotropic elastic constants \\
\hline$Q_{x}, Q_{\theta}:$ & $\begin{array}{l}\text { Orthotropic elastic constants in axial and } \\
\text { circumferential direction }\end{array}$ \\
\hline$u_{0}, v_{0}, w_{0}:$ & Displacement in $x, y, z$ directions \\
\hline$\dot{u}_{0}, \dot{v}_{0}, \dot{w}_{0}:$ & Velocity in $x, y, z$ directions \\
\hline$U:$ & Virtual strain energy \\
\hline$U_{\max }:$ & Maximum strain energy \\
\hline$V_{\mathrm{CN}}, V_{\mathrm{CN}}^{*}:$ & Volume fractions of carbon nanotubes \\
\hline$V_{m}:$ & $\begin{array}{l}\text { Volume fractions related to the back- } \\
\text { ground }\end{array}$ \\
\hline$w_{\mathrm{CN}}:$ & Mass fraction of carbon nanotubes \\
\hline$x:$ & $\begin{array}{l}\text { Coordinate axis in the ridge direction of } \\
\text { the cone }\end{array}$ \\
\hline$z:$ & Centerline of the cone \\
\hline$\beta:$ & Cone vertex angle \\
\hline$\Pi:$ & Energy functional \\
\hline$\widehat{\Pi}:$ & Maximum energy functional \\
\hline$\varepsilon_{x x}, \varepsilon_{\theta \theta}, \gamma_{x \theta}$ & $\begin{array}{l}\text { Strain-displacement relations for an arbi- } \\
\text { trary point in the distance } z \text { from the } \\
\text { middle surface }\end{array}$ \\
\hline$\varepsilon_{x x}^{0}, \varepsilon_{\theta \theta}^{0}, \gamma_{x \theta}^{0}$ & $\begin{array}{l}\text { Linear strain-displacement relations of } \\
\text { the middle surface }\end{array}$ \\
\hline$\theta:$ & Coordinate in environmental direction \\
\hline$\phi:$ & Half cone angle \\
\hline$\sigma_{x x}, \sigma_{\theta \theta}, \sigma_{x \theta}:$ & Stress components \\
\hline$v_{i j}:$ & Poisson's ratio \\
\hline$v_{i j}^{\mathrm{CN}}:$ & Poisson's ratio of carbon nanotubes \\
\hline$\nu^{m}:$ & Poisson's ratio related to the background \\
\hline$\eta_{j}:$ & Efficiency parameter of carbon nanotubes \\
\hline$\omega:$ & Natural frequency \\
\hline$\rho:$ & Mass density \\
\hline$\rho_{\mathrm{CN}}:$ & Mass density of carbon nanotubes \\
\hline$\rho^{m}:$ & Mass density related to the background. \\
\hline
\end{tabular}

\section{Competing Interests}

The authors declare that they have no competing interests.

\section{References}

[1] C. Yeh, M. Chen, J. Hwang, J. Gan, and C. Kou, "Field emission from a composite structure consisting of vertically aligned single-walled carbon nanotubes and carbon nanocones," Nanotechnology, vol. 17, pp. 5930-5935, 2006.

[2] A. Koochi, A. S. Kazemi, A. Noghrehabadi, A. Yekrangi, and M. Abadyan, "New approach to model the buckling and stable length of multi walled carbon nanotube probes near graphite sheets," Materials and Design, vol. 32, no. 5, pp. 2949-2955, 2011.

[3] A. Koochi, A. Kazemi, and M. Abadyan, "Simulating deflection and determining stable length of freestanding carbon nanotube probe/sensor in the vicinity of graphene layers using a nanoscale continuum model," Nano, vol. 6, no. 5, pp. 419-429, 2011.

[4] S. L. Das, T. Mandal, and S. S. Gupta, "Inextensional vibration of zig-zag single-walled carbon nanotubes using nonlocal elasticity theories," International Journal of Solids and Structures, vol. 50, no. 18, pp. 2792-2797, 2013.
[5] B. Fang, Y.-X. Zhen, C.-P. Zhang, and Y. Tang, "Nonlinear vibration analysis of double-walled carbon nanotubes based on nonlocal elasticity theory," Applied Mathematical Modelling, vol. 37, no. 3, pp. 1096-1107, 2013.

[6] Y. Huang, Q.-Z. Luo, and X.-F. Li, “Transverse waves propagating in carbon nanotubes via a higher-order nonlocal beam model," Composite Structures, vol. 95, pp. 328-336, 2013.

[7] K. Kiani, "Vibration behavior of simply supported inclined single-walled carbon nanotubes conveying viscous fluids flow using nonlocal Rayleigh beam model," Applied Mathematical Modelling, vol. 37, no. 4, pp. 1836-1850, 2013.

[8] B. L. Wang and K. F. Wang, "Vibration analysis of embedded nanotubes using nonlocal continuum theory," Composites Part B: Engineering, vol. 47, pp. 96-101, 2013.

[9] Y. Tadi Beni, F. Mehralian, and H. Razavi, "Free vibration analysis of size-dependent shear deformable functionally graded cylindrical shell on the basis of modified couple stress theory," Composite Structures, vol. 120, pp. 65-78, 2015.

[10] S. Sahmani, R. Ansari, R. Gholami, and A. Darvizeh, "Dynamic stability analysis of functionally graded higher-order shear deformable microshells based on the modified couple stress elasticity theory," Composites Part B: Engineering, vol. 51, pp. 4453, 2013.

[11] M. Simsek and J. N. Reddy, "Bending and vibration of functionally graded microbeams using a new higher order beam theory and the modified couple stress," International Journal of Engineering Science, vol. 64, pp. 37-53, 2013.

[12] Y. Tadi Beni, A. Koochi, and M. Abadyan, "Theoretical study of the effect of Casimir force, elastic boundary conditions and size dependency on the pull-in instability of beam-type NEMS," Physica E: Low-Dimensional Systems and Nanostructures, vol. 43, no. 4, pp. 979-988, 2011.

[13] L. Wang, Y. Y. Xu, and Q. Ni, "Size-dependent vibration analysis of three-dimensional cylindrical microbeams based on modified couple stress theory: a unified treatment," International Journal of Engineering Science, vol. 68, pp. 1-10, 2013.

[14] R. Ansari, R. Gholami, M. Faghih Shojaei, V. Mohammadi, and S. Sahmani, "Size-dependent bending, buckling and free vibration of functionally graded Timoshenko microbeams based on the most general strain gradient theory," Composite Structures, vol. 100, pp. 385-397, 2013.

[15] A. G. Arani, A. R. Shajari, S. Amir, and A. Loghman, "Electrothermo-mechanical nonlinear nonlocal vibration and instability of embedded micro-tube reinforced by BNNT, conveying fluid," Physica E, vol. 45, pp. 109-121, 2012.

[16] S. Sahmani and R. Ansari, "On the free vibration response of functionally graded higher-order shear deformable microplates based on the strain gradient elasticity theory," Composite Structures, vol. 95, pp. 430-442, 2013.

[17] M. E. Gurtin, J. Weissmüller, and F. Larché, "A general theory of curved deformable interfaces in solids at equilibrium," Philosophical Magazine A: Physics of Condensed Matter, Structure, Defects and Mechanical Properties, vol. 78, no. 5, pp. 1093-1109, 1998.

[18] A. Koochi, A. Kazemi, F. Khandani, and M. Abadyan, "Influence of surface effects on size-dependent instability of nanoactuators in the presence of quantum vacuum fluctuations," Physica Scripta, vol. 85, no. 3, Article ID 035804, 2012.

[19] A. Koochi, H. Hosseini-Toudeshky, H. R. Ovesy, and M. Abadyan, "Modeling the influence of surface effect on instability of nano-cantilever in presence of Van der Waals force," International Journal of Structural Stability and Dynamics, vol. 13, no. 4, Article ID 250072, 2013. 
[20] Y. T. Beni, A. Koochi, A. S. Kazemi, and M. Abadyan, "Modeling the influence of surface effect and molecular force on pull-in voltage of rotational nano-micro mirror using 2-DOF model," Canadian Journal of Physics, vol. 90, no. 10, pp. 963-974, 2012.

[21] S. Shayan-Amin, H. Dalir, and A. Farshidianfar, "Molecular dynamics simulation of double-walled carbon nanotube vibrations: comparison with continuum elastic theories," Journal of Mechanics, vol. 25, no. 4, pp. 337-343, 2009.

[22] W. H. Duan, C. M. Wang, and Y. Y. Zhang, "Calibration of nonlocal scaling effect parameter for free vibration of carbon nanotubes by molecular dynamics," Journal of Applied Physics, vol. 101, no. 2, Article ID 024305, 2007.

[23] R. Maranganti and P. Sharma, "A novel atomistic approach to determine strain-gradient elasticity constants: tabulation and comparison for various metals, semiconductors, silica, polymers and the (Ir) relevance for nanotechnologies," Journal of the Mechanics and Physics of Solids, vol. 55, no. 9, pp. 18231852, 2007.

[24] J. H. Lee and B. S. Lee, "Modal analysis of carbon nanotubes and nanocones using FEM," Computational Materials Science, vol. 51, no. 1, pp. 30-42, 2012.

[25] D. C. C. Lam, F. Yang, A. C. M. Chong, J. Wang, and P. Tong, "Experiments and theory in strain gradient elasticity", Journal of the Mechanics and Physics of Solids, vol. 51, no. 8, pp. 14771508, 2003.

[26] J. X. Wei, K. M. Liew, and X. Q. He, "Mechanical properties of carbon nanocones," Applied Physics Letters, vol. 91, no. 26, Article ID 261906, 2007.

[27] D. Kumar, V. Verma, H. S. Bhatti, and K. Dharamvir, "Elastic moduli of carbon nanohorns," Journal of Nanomaterials, vol. 2011, Article ID 127952, 6 pages, 2011.

[28] J. W. Yan, K. M. Liew, and L. H. He, "Buckling and post-buckling of single-wall carbon nanocones upon bending," Composite Structures, vol. 106, pp. 793-798, 2013.

[29] M. Ezrin, Plastics Failure Guide: Cause and Prevention, Hanser, 1996.

[30] R. A. Meyers, Molecular Biology and Biotechnology: A Comprehensive Desk Reference, Wiley-VCH, 1995.

[31] J. Y. Rho, R. B. Ashman, and C. H. Turner, "Young's modulus of trabecular and cortical bone material: ultrasonic and microtensile measurements," Journal of Biomechanics, vol. 26, no. 2, pp. 111-119, 1993.

[32] M. D. Miller, Review of Orthopedics, W. B. Saunders, Philadelphia, Pa, USA, 4th edition, 1996.

[33] L.-L. Ke, J. Yang, and S. Kitipornchai, "Nonlinear free vibration of functionally graded carbon nanotube-reinforced composite beams," Composite Structures, vol. 92, no. 3, pp. 676-683, 2010.

[34] R. Ansari, M. F. Shojaei, V. Mohammadi, R. Gholami, and F. Sadeghi, "Nonlinear forced vibration analysis of functionally graded carbon nanotube-reinforced composite Timoshenko beams," Composite Structures, vol. 113, no. 1, pp. 316-327, 2014.

[35] R. Ansari, E. Hasrati, M. Faghih Shojaei, R. Gholami, and A. Shahabodini, "Forced vibration analysis of functionally graded carbon nanotube-reinforced composite plates using a numerical strategy," Physica E: Low-Dimensional Systems and Nanostructures, vol. 69, pp. 294-305, 2015.

[36] R. Ansari and R. Gholami, "Nonlinear primary resonance of third-order shear deformable functionally graded nanocomposite rectangular plates reinforced by carbon nanotubes," Composite Structures, vol. 154, pp. 707-723, 2016.

[37] L. W. Zhang and K. M. Liew, "Geometrically nonlinear large deformation analysis of functionally graded carbon nanotube reinforced composite straight-sided quadrilateral plates," Computer Methods in Applied Mechanics and Engineering, vol. 295, pp. 219-239, 2015.

[38] Z. X. Lei, L. W. Zhang, and K. M. Liew, "Elastodynamic analysis of carbon nanotube-reinforced functionally graded plates," International Journal of Mechanical Sciences, vol. 99, pp. 208-217, 2015.

[39] R. Ansari, T. Pourashraf, R. Gholami, and A. Shahabodini, "Analytical solution for nonlinear postbuckling of functionally graded carbon nanotube-reinforced composite shells with piezoelectric layers," Composites Part B: Engineering, vol. 90, pp. 267-277, 2016.

[40] R. Ansari and J. Torabi, "Numerical study on the buckling and vibration of functionally graded carbon nanotube-reinforced composite conical shells under axial loading," Composites Part B: Engineering, vol. 95, pp. 196-208, 2016.

[41] R. Ansari, J. Torabi, M. Faghih Shojaei, and E. Hasrati, "Buckling analysis of axially-loaded functionally graded carbon nanotubereinforced composite conical panels using a novel numerical variational method," Composite Structures, vol. 157, pp. 398-411, 2016.

[42] K. M. Liew, T. Y. Ng, and X. Zhao, "Free vibration analysis of conical shells via the element-free kp-Ritz method," Journal of Sound and Vibration, vol. 281, no. 3-5, pp. 627-645, 2005.

[43] X. Zhao and K. M. Liew, "Free vibration analysis of functionally graded conical shell panels by a meshless method," Composite Structures, vol. 93, no. 2, pp. 649-664, 2011.

[44] G. Formica, W. Lacarbonara, and R. Alessi, "Vibrations of carbon nanotube-reinforced composites," Journal of Sound and Vibration, vol. 329, no. 10, pp. 1875-1889, 2010.

[45] T. Mori and K. Tanaka, "Average stress in matrix and average elastic energy of materials with misfitting inclusions," Acta Metallurgica, vol. 21, no. 5, pp. 571-574, 1973.

[46] J. D. Eshelby, "The determination of the elastic field of an ellipsoidal inclusion, and related problems," Proceedings of the Royal Society of London A: Mathematical, Physical and Engineering Sciences, vol. 241, pp. 376-396, 1957.

[47] F. Tornabene, "Free vibration analysis of functionally graded conical, cylindrical shell and annular plate structures with a four-parameter power-law distribution," Computer Methods in Applied Mechanics and Engineering, vol. 198, no. 37-40, pp. 29112935, 2009.

[48] K. K. Viswanathan and P. V. Navaneethakrishnan, "Free vibration of layered truncated conical shell frusta of differently varying thickness by the method of collocation with cubic and quintic splines," International Journal of Solids and Structures, vol. 42, no. 3-4, pp. 1129-1150, 2005.

[49] Y. Han and J. Elliott, "Molecular dynamics simulations of the elastic properties of polymer/carbon nanotube composites," Computational Materials Science, vol. 39, no. 2, pp. 315-323, 2007.

[50] H.-S. Shen, "Nonlinear bending of functionally graded carbon nanotube-reinforced composite plates in thermal environments," Composite Structures, vol. 91, no. 1, pp. 9-19, 2009.

[51] H. Fukuda and K. Kawata, "On Young's modulus of short fibre composites," Fibre Science and Technology, vol. 7, no. 3, pp. 207$222,1974$. 

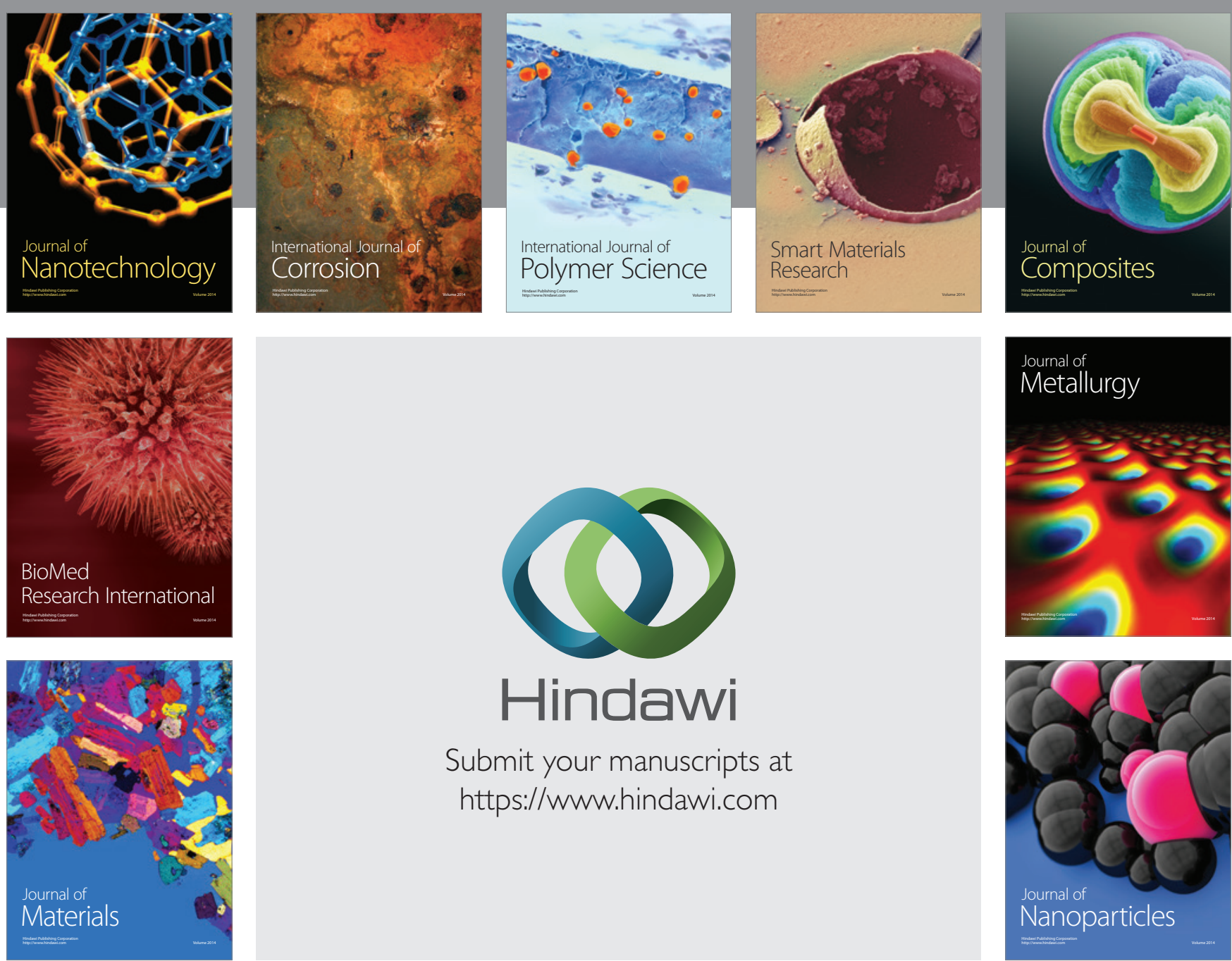

\section{Hindawi}

Submit your manuscripts at

https://www.hindawi.com

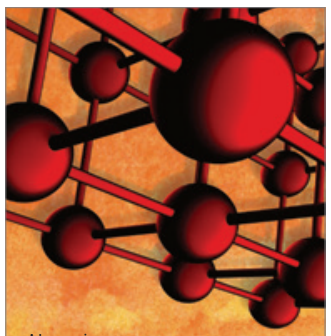

Materials Science and Engineering
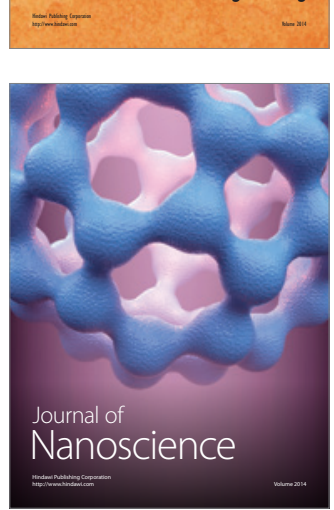
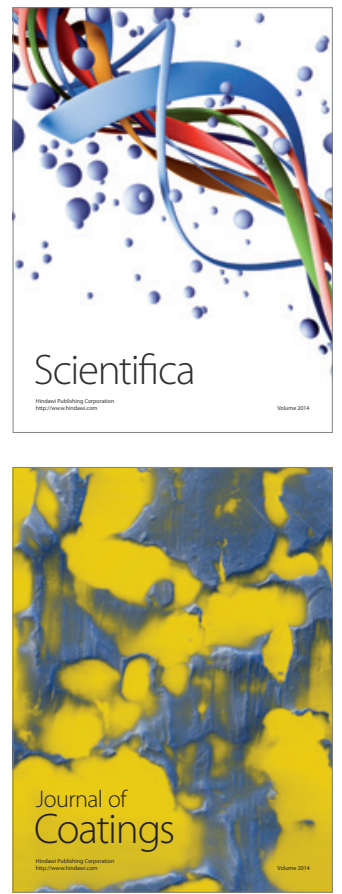
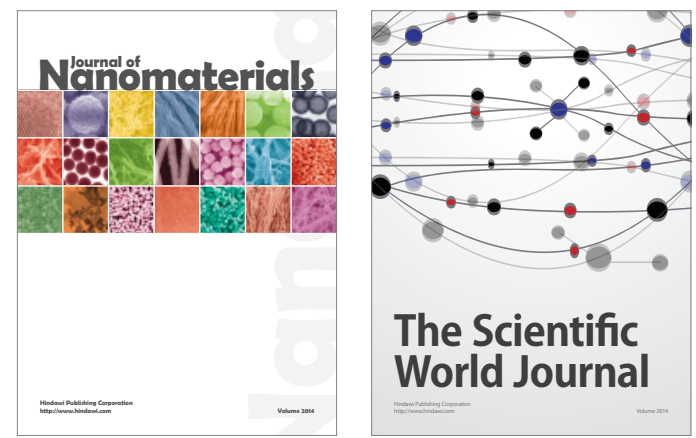

The Scientific World Journal
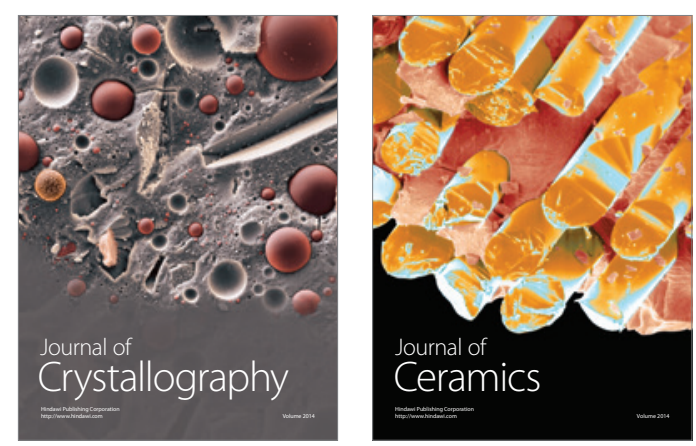
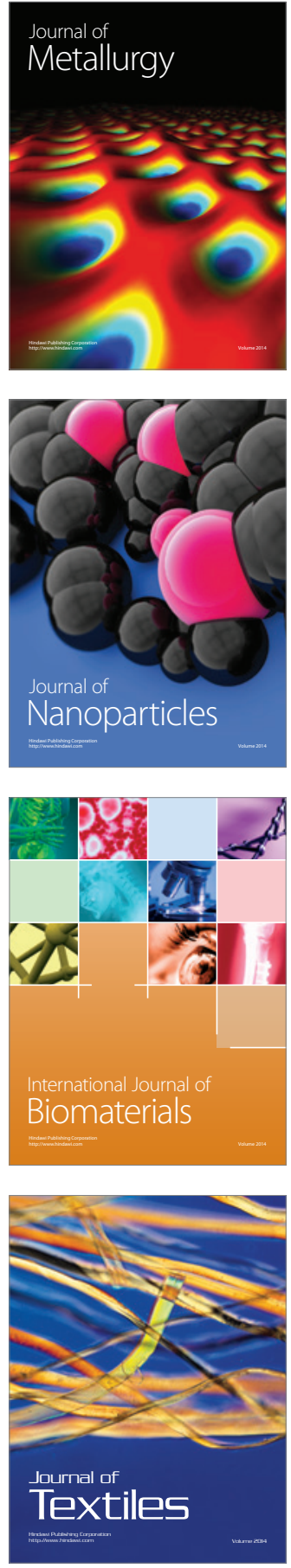